\title{
The dark side of alpha-particle optical potential: Emission from excited nuclei
}

\author{
Vlad Avrigeanu* and Marilena Avrigeanu \\ ${ }^{1}$ Horia Hulubei National Institute for Physics and Nuclear Engineering, P.O. Box MG-6, 077125 Bucharest-Magurele, Romania
}

\begin{abstract}
Further analysis of $\alpha$-particle emission from similar nuclei excited in neutron- as well as low-energy proton-induced reactions is involved concerning a possible difference between the optical model potentials (OMPs) which describe either alpha-particle elastic scattering and induced reactions or alpha-emission from excited compound nuclei. The key role of the pickup direct interaction is finally proved even at incident energies below $\sim 10 \mathrm{MeV}$, as well as being eventually at the origin of the above-mentioned OMPs difference.
\end{abstract}

\section{Introduction}

The $\alpha$-particle interaction with nuclei and the corresponding optical model potential (OMP) were of special interest from the earliest days of nuclear physics. The widely-used phenomenological OMP parameters were derived from analysis of either elastic-scattering or $\alpha$-induced reaction data and then used to describe also the $\alpha$-particle emission from hot nuclei excited in nuclear reactions. However the later studies are also subject of various assumptions and quantities. Thus, the $\alpha$-particle OMP for the incident channel seems similar to the familiar side of the Moon which is facing always the Earth, but for the $\alpha$-emission it is like the dark side of the Moon. Moreover, there is a so-called $\alpha$-potential mystery [1] of the account at once of both absorption and emission of alpha particles, of equal interest for nuclear astrophysics and technology.

A former search for new physics in potentials to describe nuclear de-excitation used the assumption that particle evaporation occurs from a transient nuclear stratosphere of the emitter nucleus ([2] and Refs. therein). However, only definite conclusion concerning the incident channel $[3,4]$ has made feasible the analysis of a real difference between the OMPs describing either $\alpha$-particle elastic scattering and induced reactions or $\alpha$-emission from excited compound nuclei [5]. Meantime, better results provided by potential [3] within large-scale nucleardata evaluation [6] led to its adoption as the latest default option within the code TALYS [7]. At the same time the OMP [3] validation for the $\alpha$-particle emission in lowenergy proton-induced reactions on $\mathrm{Zn}$ isotopes [8] has been related to a surface character of these reactions, at possible variance to the fast-neutron induced reactions on $\mathrm{Zr}$ isotopes [9]. Further analysis of $\alpha$-particle emission from similar $\mathrm{Co}, \mathrm{Cu}$, and $\mathrm{Zn}$ nuclei excited in neutron- as well as low-energy proton-induced reactions could make clear these points and makes the object of this work.

Hauser-Feshbach (HF) statistical model and Geometry-Dependent Hybrid (GDH) [10] pre-equilibrium

\footnotetext{
*e-mail: vlad.avrigeanu@nipne.ro
}

emission (PE) calculations have been carried out in this respect using an updated version of the code STAPREH95 [11], with $0.2-0.3 \mathrm{MeV}$ equidistant binning of the excitation energy grid. The direct interaction (DI) distorted-wave Born approximation (DWBA) method was involved within the code FRESCO [12] for calculation of collective inelastic scattering and pickup reactions cross sections. These results were also involved to subsequently decrease the total-reaction cross section $\sigma_{R}$ within the $\mathrm{PE}+\mathrm{HF}$ calculations. The prior form DW transition amplitudes and the finite-range interaction were considered similar to recent deuteron-induced reaction studies ([13] and Refs. therein).

Last but not least, no empirical rescaling factors of the $\gamma$ and/or neutron widths were used but consistent parameter sets given elsewhere $[3,4,8,9]$. Results of this work are compared with the evaluated data library TENDL2017 [14], for an overall excitation function survey.

\section{$2 \alpha$-emission from $\mathrm{Cu}$ excited nuclei}

A previous consistent analysis of ${ }^{63,65} \mathrm{Cu}$ neutron activation [15] already revealed an $\alpha$-emission overestimation

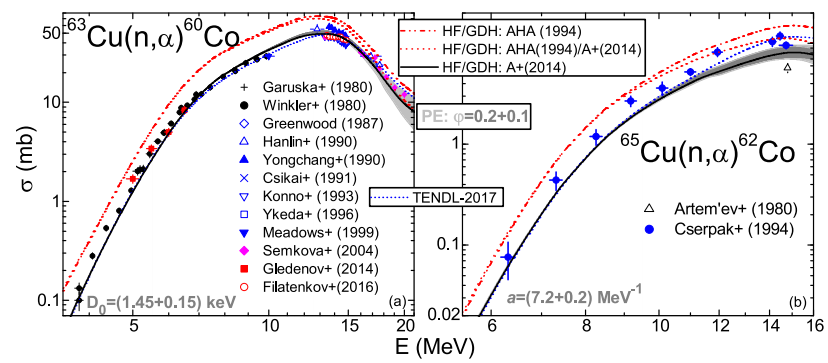

Figure 1. Comparison of measured [16] cross sections of ${ }^{63,65} \mathrm{Cu}(n, \alpha)$ reactions with TENDL-2017 evaluation [14] (shortdotted) and PE+HF calculations using $\alpha$-particle OMPs [5] (dash-dot-dotted), [3] (solid), and both (dotted), with uncertainty bands for NLD (light gray) and PE (gray) effects on [3] results. 
by the OMP [5]. It has been strengthened by the accurate measurements performed in the meantime especially on ${ }^{63} \mathrm{Cu}$ at low energies. The OMP [3] provides however calculated cross sections in a rather good agreement especially with these data at energies where nuclear-level density (NLD) and PE effects shown in Fig. 1 by the related uncertainty bands (e.g., Ref. [4]b) are low.

On the other hand, the $\alpha$-emission leading also to a ${ }^{61} \mathrm{Co}$ isotope but in $(p, \alpha)$ reaction made the object of the pioneering work of Qaim et al. [17]. Actually they extended for the first time at incident energies $<10 \mathrm{MeV}$ the conclusion of ' 90 s that, at least for low energies as well as DI contributions to the low-lying levels, the main mechanism in $(n / p, \alpha)$ reactions is pickup instead of knockout ([18] and Refs. therein). This was considered by them for $\mathrm{PE}+\mathrm{HF}$ underestimation for ${ }^{64} \mathrm{Ni}(p, \alpha){ }^{61}$ Co reaction, get also in this work at once with suitable account of even newest data for other channels (Fig. 2) using the same consistent parameter set of ${ }^{63,65} \mathrm{Cu}(n, \alpha){ }^{60,62} \mathrm{Co}$ analysis.

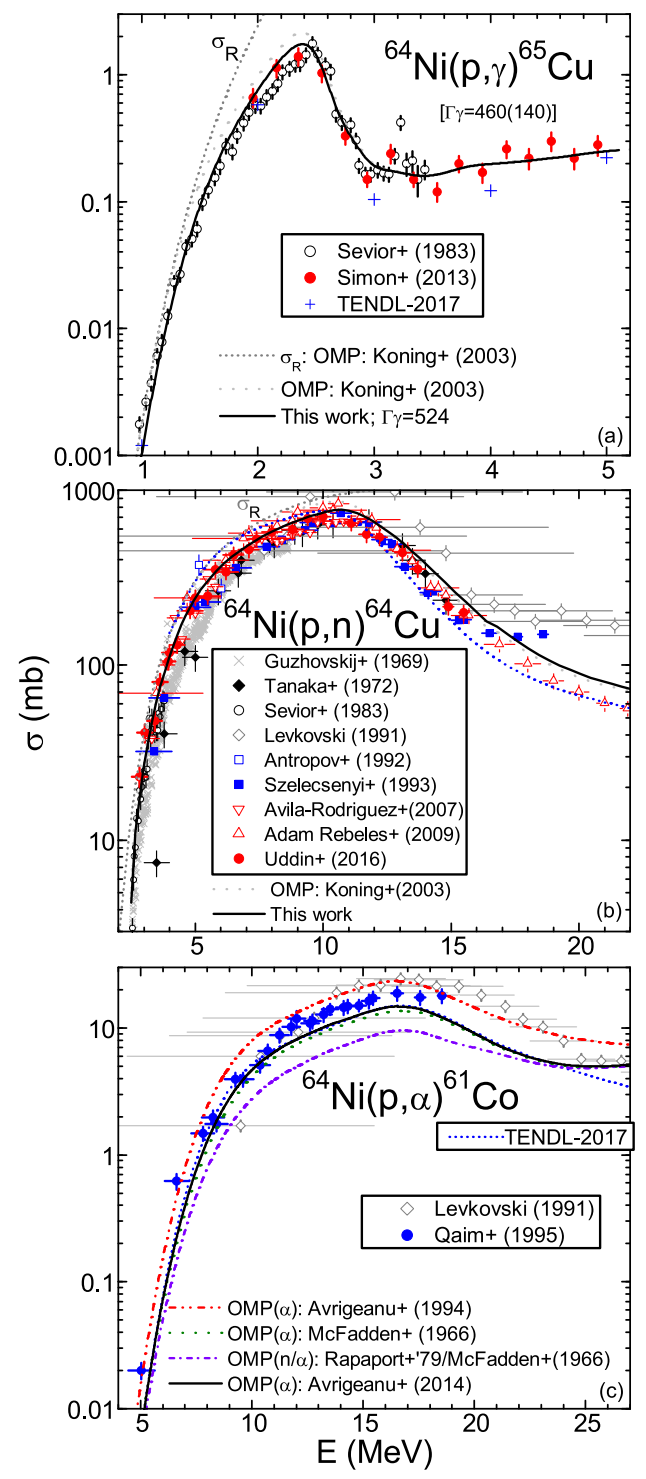

Figure 2. As Fig. 1 but for ${ }^{64} \mathrm{Ni}(p, x)$ reactions, except the use of OMPs [19] (a,b) and [20, 21] (c) (dotted).

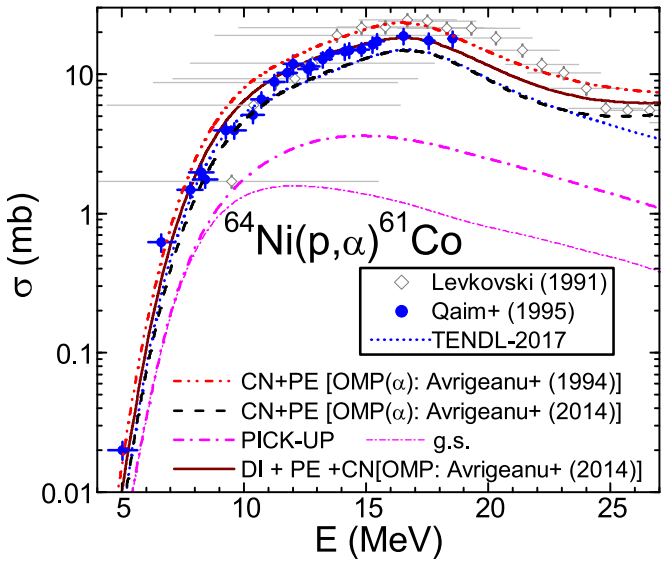

Figure 3. As Fig. 1 but for ${ }^{64} \mathrm{Ni}(p, \alpha){ }^{61} \mathrm{Co}$ reaction, except additional g.s. (thin dash-dotted) and total (dash-dotted) DI components of total cross sections (solid).

The lack of independent experimental data led Qaim et al. to normalize the pickup contribution in order to describe their $(p, \alpha)$ data at $15 \mathrm{MeV}$ [17]. However, this normalization depends notably on the former PE+HF calculated results. Since these are quite different in the case of the OMPs involved by Qaim et al. and us, respectively [Fig. 2(c)], we have looked for absolute values by DWBA analysis using spectroscopic factors corresponding to the outgoing $\alpha$-particle angular distributions reported by Smits et al. [22]. The addition of this component provides a good account of the measured data, with a particular emphasize of the residual nucleus ground-state (g.s.) population at the lowest energies (Fig. 3).

\section{$3 \alpha$-emission from $\mathrm{Zn}$ excited nuclei}

The same consistent parameter set used recently within analysis of $(\alpha, x)$ reactions on stable $\mathrm{Ni}$ isotopes below the Coulomb barrier [4] leads also to a rather good agreement for ${ }^{63,65} \mathrm{Cu}(p, \alpha)^{60,62} \mathrm{Ni}$ reactions (Fig. 4). On the other hand, a similar agreement for the more recent data of ${ }^{64,67} \mathrm{Zn}(n, \alpha)^{61,64} \mathrm{Ni}$ reactions becomes a real challenge. Thus, the same parameter set involved in the former analysis of $(\alpha, x)$ and $(p, \alpha)$ reactions provides a suitable account of the competitive channels $(n, p)$ and $(n, 2 n)$ [Fig. 5(a,b), Fig. 7(a)] while a major underestimation results for the $(n, \alpha)$ channels. It includes also the cross-section uncer-

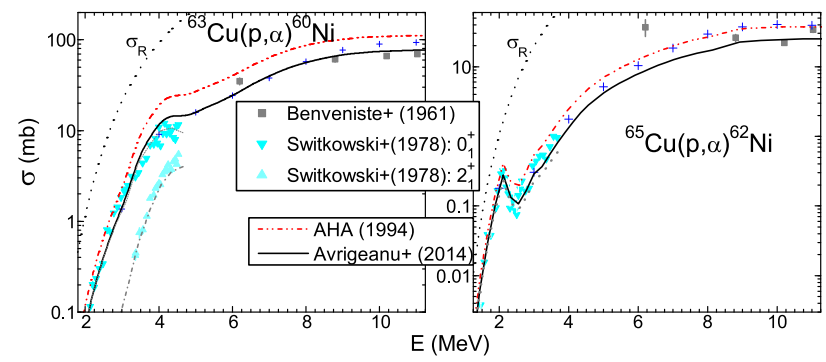

Figure 4. As Fig. 1 but for ${ }^{63,65} \mathrm{Cu}(p, \alpha)^{60,62} \mathrm{Ni}$ reactions. 
tainty bands for neutron OMP [23, 24], NLD, and PE effects [Fig. 5(c)], of the results obtained by using OMP [3].

The pickup contribution proves thus to be essential also in this case. DWBA calculated cross sections of ${ }^{64} \mathrm{Zn}(n, \alpha){ }^{61} \mathrm{Ni}$ reaction have been obtained by means of the 'spectator model' [22] for the two transferred protons, while the removing-neutron spectroscopic factors for 19 levels, up to the excitation energy of $3.308 \mathrm{MeV}$ [25], have been involved in the absence of any experimental angular distribution. The addition of this DI component is able to bring in agreement the calculated cross sections with the average trend of the measured data, at the cost of lesser account for a seeming shoulder of data as well as PE+HF results around the incident energy of 5-6 MeV (Fig. 6). Actually this shoulder is particularly shown by the partial data for the g.s. and first two excited levels of ${ }^{61} \mathrm{Ni}$ [26], with a trend well described only by the HF component.

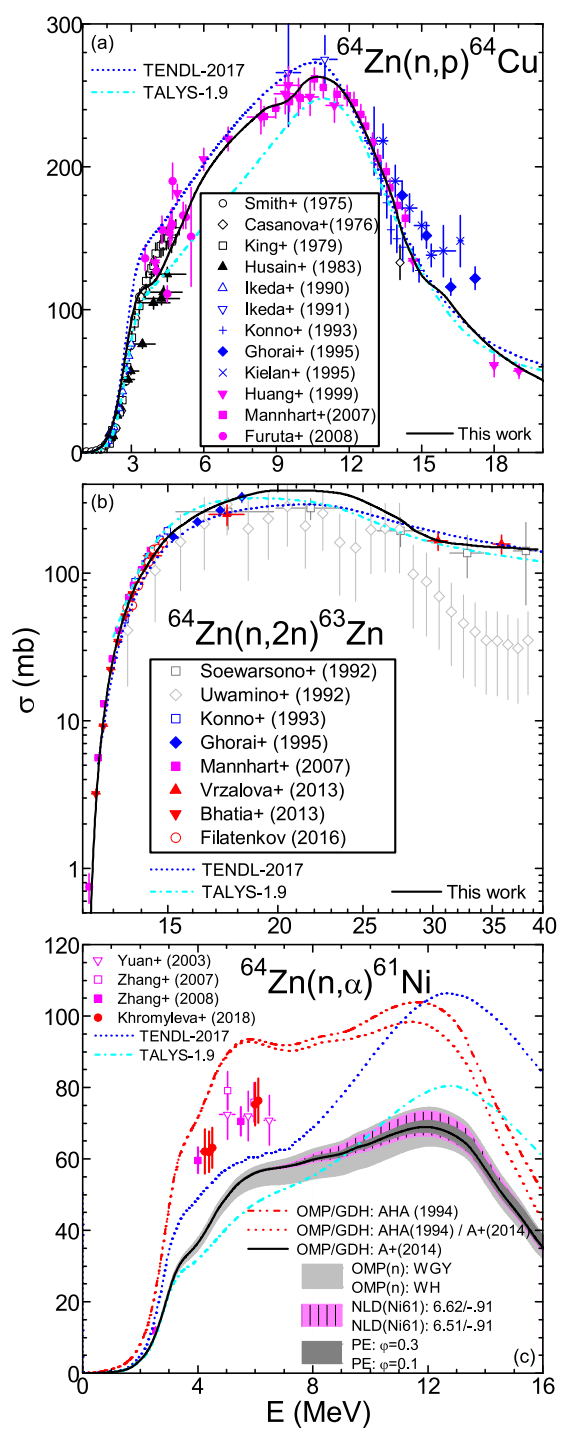

Figure 5. As Fig. 2 but for ${ }^{64} \mathrm{Zn}(n, x)$ reactions, except additional TALYS-1.9 results (short dash-dotted), and uncertainty bands for neutron OMP [23, 24] (light gray), NLD (magenta), and PE (gray) effects on the results obtained by using the OMP [3].

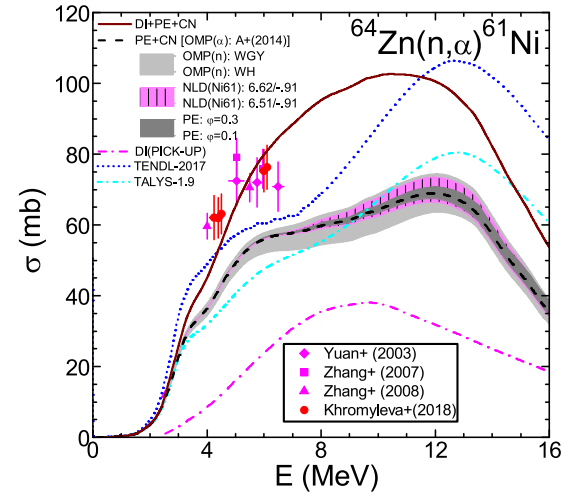

Figure 6. As Fig. 5 but for ${ }^{64} \mathrm{Zn}(n, \alpha)^{61} \mathrm{Ni}$ reaction, except additional DI component (dash-dotted) of total cross sections (solid).

Because no spectroscopic factors are available for ${ }^{64} \mathrm{Ni}$ levels, the following approximation was involved to roughly estimate the DI contribution to ${ }^{67} \mathrm{Zn}(n, \alpha){ }^{64} \mathrm{Ni}$ cross sections. The same ratio of this contribution to that of $\mathrm{PE}+\mathrm{HF}$ component is assumed for residual nuclei ${ }^{61,64} \mathrm{Ni}$. The results thus obtained [Fig. 7(c)] show a better agreement even with accurate measured partial data [27].

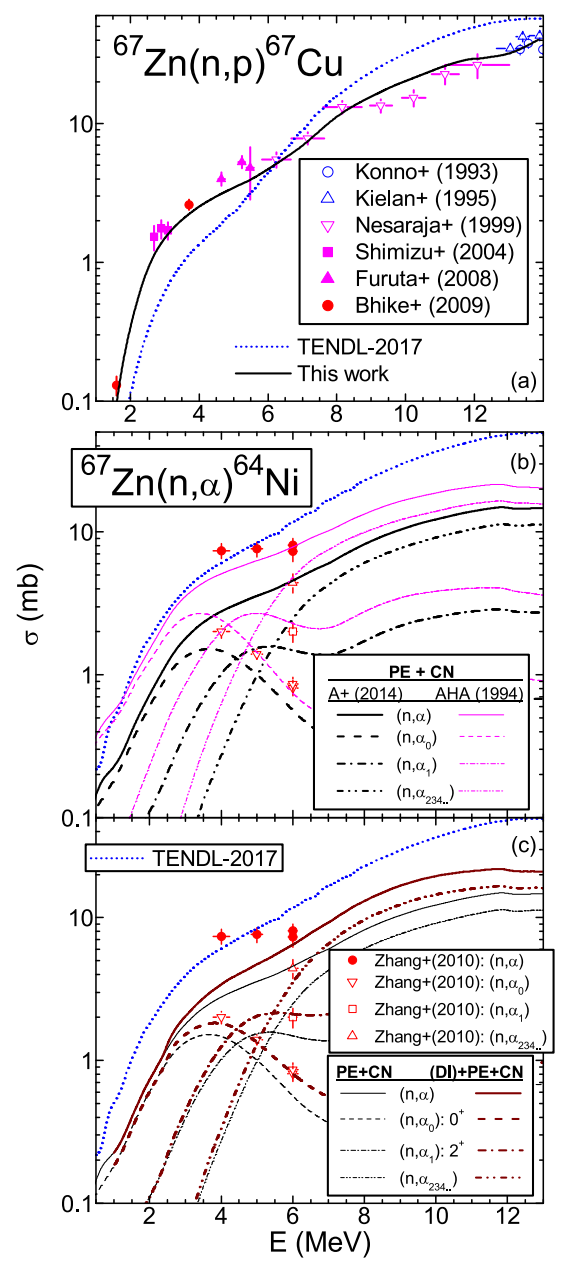

Figure 7. (a,b) As Fig. 5 but for ${ }^{67} \mathrm{Zn}(n, x)$, and (c) as Fig. 3 but for $\left.{ }^{67} \mathrm{Zn}(n, \alpha)\right)^{64} \mathrm{Ni}$ reactions, including partial components [27]. 


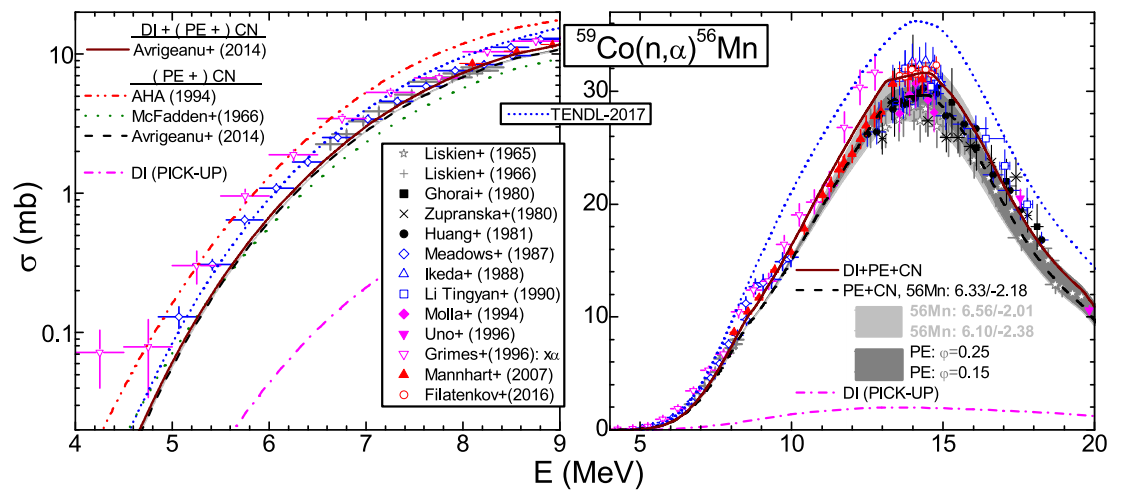

Figure 8. As Fig. 6 but for ${ }^{59} \operatorname{Co}(n, \alpha){ }^{56} \mathrm{Mn}$ reaction.

\section{$4 \alpha$-emission from Co excited nuclei}

Similar results have been obtained for ${ }^{59} \operatorname{Co}(n, \alpha)^{56} \mathrm{Mn}$ reaction (Fig. 8), with the pickup component obtained by using the neutron spectroscopic factors of Comfort [28].

\section{Conclusion}

The key role of the pickup direct interaction is proved even at incident energies $<10 \mathrm{MeV}$, as well as being eventually at the origin of the difference between OMPs which describe either alpha-particle elastic scattering and induced reactions or alpha-emission from excited nuclei.

Work within framework of Autoritatea Nationala pentru Cercetare Stiintifica (Project PN-19060102) and EUROfusion Consortium and has received funding from the Euratom research and training program 2014-2018 and 2019-2020 under grant agreement No. 633053. The views and opinions expressed herein do not necessarily reflect those of the European Commission.

\section{References}

[1] T. Rauscher, Phys. Rev. Lett. 111, 061104 (2013)

[2] M. Avrigeanu, W. von Oertzen, and V. Avrigeanu, Nucl. Phys. A 764, 246 (2006)

[3] V. Avrigeanu, M. Avrigeanu, and C. Mănăilescu, Phys. Rev. C 90, 044612 (2014)

[4] V. Avrigeanu and M. Avrigeanu, Phys. Rev. C 94, 024621 (2016); ibid 99, 044613 (2019)

[5] V. Avrigeanu, P.E. Hodgson, and M. Avrigeanu, Phys. Rev. C 49, 2136 (1994)

[6] A.J. Koning, Report EFFDOC-1271, OECD/NEA Data Bank, Paris, Nov. 2015

[7] A.J. Koning, S. Hilaire, and M. C. Duijvestijn, TALYS-1.9, Dec. 2017; http://www.talys.eu

[8] V. Avrigeanu and M. Avrigeanu, Phys. Rev. C 91, 064611 (2015)
[9] V. Avrigeanu and M. Avrigeanu, Phys. Rev. C 94, 024621 (2017)

[10] M. Blann and H.K. Vonach, Phys. Rev. C 28, 1475 (1983); M. Blann, Nucl. Phys. A 213, 570 (1973)

[11] M. Avrigeanu and V. Avrigeanu 1995 IPNE Report NP-86-1995, Bucharest, and Refs. therein, http://www.oecdnea.org/tools/abstract/detail/iaea0971/; Comp. Phys. Comm. 112, 191 (1998); A.Harangozo et al., Phys. Rev. C 58, 295 (1998)

[12] I.J. Thompson, Comput. Phys. Rep. 7, 167 (1988), v. FRES 2.9 (2011).

[13] M. Avrigeanu, C. Costache, and V. Avrigeanu, ND2019

[14] A.J. Koning and D. Rochman, https://tendl.web.psi.ch/tendl_2017/tend12017.html

[15] M. Avrigeanu et al., Nucl. Phys. A 806, 15 (2008)

[16] Experimental Nuclear Reaction Data (EXFOR), https://www-nds.iaea.org/exfor/

[17] S.M. Qaim, M. Uhl, F. Rösch, and F. Szelecsényi, Phys. Rev. C 52, 733 (1995)

[18] E. Gadioli and P.E. Hodgson, Pre-Equilibrium Nuclear Reactions (Clarendon, Oxford, 1992)

[19] A.J. Koning and J.P. Delaroche, Nucl. Phys. A713, 231 (2003)

[20] L. McFadden and G.R. Satchler, Nucl. Phys. A84, 177 (1966)

[21] J. Rapaport, V. Kulkarni, and R.W. Finlay, Nucl. Phys. A 330, 15 (1979)

[22] J.W. Smits et al., Nucl. Phys. A 319, 29 (1979)

[23] D. Wilmore and P.E. Hodgson, Nucl. Phys. 55, 673 (1964)

[24] N. Yamamuro, Int.Conf.on NDST, Mito, 1988, p.489

[25] J.P. Schiffer et al., Phys. Rev. C 87, 034306 (2013)

[26] Guohui Zhang et al., Nucl. Sci. Eng. 156, 115 (2007); ibid 160, 123 (2008)

[27] Guohui Zhang et al., Phys. Rev. C 82, 054619 (2010)

[28] J.R. Comfort, Phys. Rev. 177, 1573 (1969) 